\title{
Shortwave Emission from Triboplasma Discharge and Its Potential Application for Light Trapping Agricultural Pest by Wind-Driven Friction
}

\author{
Xiang-Yang WANG ${ }^{1, a}$, Jian-Chao WANG ${ }^{2, b}$ and Qiang Z ZHOU ${ }^{3, c, *}$ \\ 1,2,3 Department of Mechatronics, College of Engineering, China Agricultural University, Beijing \\ 100083, China \\ ayangfischer@163.com b wjianchao@cau.edu.cn c c*zq@cau.edu.cn
}

\begin{abstract}
Keywords: Shortwave Emission, Triboplasma Discharge, Light Trapping, Agricultural Pests Control, Wind-Driving.
\end{abstract}

\begin{abstract}
High intensive shortwave light can be obtained through triboelectrification induced triboplasma discharge in sliding friction. Therefore, It will present a new method for emitting shortwave light which can be used as light source to control agricultural pests by wind-driven sliding friction without extra energy supply. The intensity of shortwave emission from triboplasma discharge induced by triboelectrification in reciprocating rubbing movement between PTFE and quartz tube filled Ar-N2 mixed gas had be investigated. The spectrum of shortwave emission near UV and violet and especially 52282 counts light intensity at 337nm peak wavelength was obtained. Further, the double-cylindrical shape of quartz tube was designed to form the triboplasma light emission device. The vertical shaft wind turbine was used to constitute the rotating driving part to satisfy the self-powered application needs.This work provides a novel method to emit shortwave emission for light trapping agricultural pests to control crop/forage grass pest-insects without pesticides by utilizing windy energy in the vast field or wild grassland.
\end{abstract}

\section{Introduction}

Previously, some triboluminescence(TL) phenomenon has been confirmed from dielectric breakdown of the surrounding air[1,2]. The spectra of light emission by TL is similar to the spectra of air discharge. Triboplasma concept was further confirmed by Keiji Nakayama through a series of investigations on electro-magnetic radiation and particles emitted from the sliding contact in vacuum, in ambient air, in various gas atmospheres[3,4]. Nakayama has proposed a mechanism of triboemission from tribomicroplasma and the light emission has been explained by the discharge of ambient gas molecules emitting ultraviolet photons through an electron avalanche process by intense electric fields caused by tribocharging or triboelectrification[5,6].

Recently, A self-powered UV light emitting device has been designed based on triboelectrification induce low pressure gas plasma discharge[7]. This device can emit $253.7 \mathrm{~nm}$ UV light only by low-frequency mechanical friction between polymer and quartz glass tube containing Ar-Hg gas. The deep UV sterilization experiment shows that the self-powered UV light emitting device can be effectively applied for sterilization.

In this work, a near UV light emission from triboplasma discharge was obtained by means of the reciprocating cycle of mechanical friction between PTFE and quartz glass tube. Shortwave light is sensitive to most insects that can be used in controlling agricultural pests without pesticides. Therefore it presents a novel design to fabricate a UV light emitting device using wind-driven mechanical friction to correlate the triboelectrification with triboplasma discharge. This self-powered device driven by wind can be useful for controlling agricultural pest insect in the vast field without extra energy supply. So it is not only a novel method of utilizing windy energy, also a new pathway of controlling crop/forage grass pest-insects in the vast field or wild grassland.

\section{Shortwave Emission from Ar-N2 Triboplasma Discharge}

\section{Theoretical and Experimental Analysis}

According to the Contact electrification, tribocharges will be generated when there is friction 
between two different dielectric materials[8]. Such as PTFE and quartz glass, those two kind of material are far away from each other on the triboelectric series[9], and the surface of two material will be charged negatively and positively respectively. Therefore, the changing electric field can be generated by the reciprocating cycle of mechanical friction the friction of PTFE on glass(see Fig 1).

Based on Mechanism of triboplasma generation, The electrons emitted from the quartz glass surface or electrons produced in a gap of the contact are accelerated by this intense electric field[10]. The accelerated electrons $\mathrm{e}^{-}$, then collide with $\mathrm{Ar}$ and $\mathrm{N}_{2}$ molecules in the gap to produce additional electrons, $e^{-}$and the excited state molecules of $N_{2}, N_{2}{ }^{*}\left(N_{2}\left(X^{1} \sum_{g}{ }^{+}\right)+A r^{*} \rightarrow N_{2}{ }^{*}\left(C^{3} \Pi_{u}\right)+A r\right)$. This process causes an electron avalanche producing an enormous number of electrons and triboplasma is generated below the surface sliding contact. When the electrons in the excited level fall down to the lower or ground level, shortwave light will be emitted $\left(\mathrm{N}_{2}{ }^{*}\left(\mathrm{C}^{3} \Pi_{\mathrm{u}}\right) \rightarrow \mathrm{N}_{2}{ }^{*}\left(\mathrm{~B}^{3} \Pi_{\mathrm{g}}\right)+\mathrm{hv}\right)$. Therefore, a mechanical rubbing of the PTFE and quartz glass filled low pressure Ar- $\mathrm{N}_{2}$ gas may yield a continuous shortwave emission.

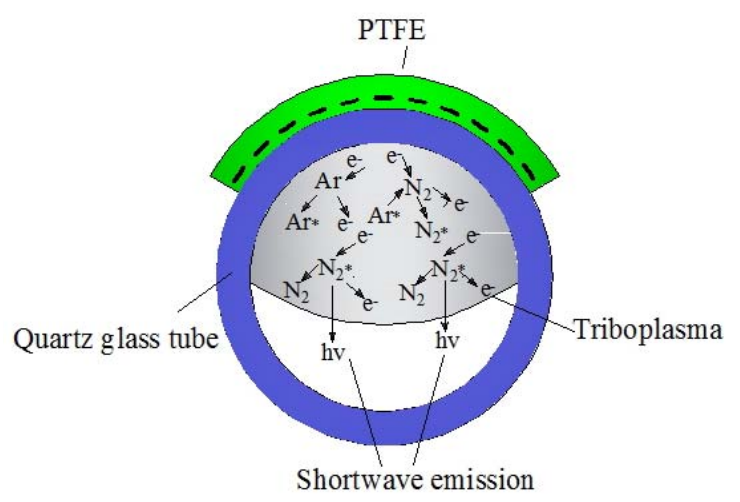

Figure 1 Principle Scheme of Shortwave Light Emission from Triboplasma Discharge

\section{Triboplasma Experimental Device}

Based on the principle scheme of gas triboplasma discharge, the experimental device of continuing shortwave light emission is constituted. As shown in figure 2, the PTFE was fixed on a platform that can impose a load of 0 to $30 \mathrm{~N}$ on the contact surface between those two materials(see Fig 2). The platform was attached to a conveyor belt drived by a AC servo motor, which could perform the continuous cycle of back-and-forth motion with the speed range from 0 to $1.5 \mathrm{~m} / \mathrm{s}$. The vacuum pump ensured a high vacuum state in the quartz glass tube, the $\mathrm{Ar}^{-\mathrm{N}_{2}}$ (mixing ratio of 1:9) is filled into the quartz glass tube to maintain a negative pressure at $30000 \mathrm{~Pa}$. A fiber probe was placed the other side of quartz tube to detect photons emission by the spectrometer (AvaSpec-2048). And all the trials were produced in a glove box in order to to avoid the influence of humidity.

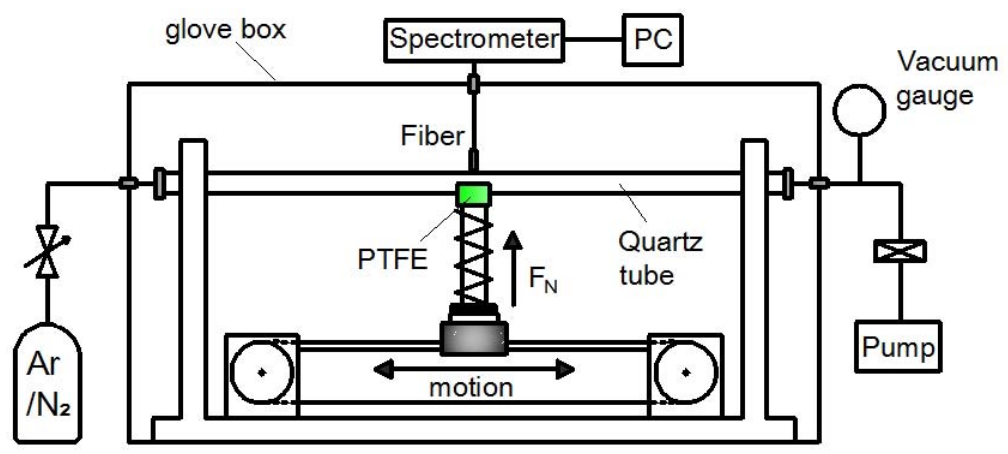

Figure 2 Schematic Representation of The Experimental Apparatus 


\section{Experimental Results}

The intensity of shortwave emission from triboplasma discharge was tested using experimental device shown in figure 2. Some important factors influence emission intensity have been investigated, including gas pressure, sliding velocity and normal force. A optimum shortwave light emission was obtained in the condition of $10 \mathrm{~N}$ applied load and $1 \mathrm{~m} / \mathrm{s}$ sliding friction velocity and low pressure Ar- $\mathrm{N}_{2}$ gas at 30000pa, as shown in figure 3. The sharp peaks in 310nm to $410 \mathrm{~nm}$ (316, $337,357,375,381,406 \mathrm{~nm})$ are emission from the second positive system of $\mathrm{N}_{2}\left(\mathrm{~N}_{2}{ }^{*}\left(\mathrm{C}^{3} \Pi_{\mathrm{u}}\right) \rightarrow\right.$ $\mathrm{N}_{2}{ }^{*}\left(\mathrm{~B}^{3} \Pi_{\mathrm{g}}\right)$ ). The sharp peaks in $680 \mathrm{~nm}$ to $800 \mathrm{~nm}$ are emission from the excited state molecule of Ar. The result shows the high intensity emission of ten thousand counts in the full near UV, even the peak value wavelength more than fifty thousand counts. It also indicates shortwave emission from $\mathrm{Ar}$-N2 gas triboplasma discharge can be used as special light source.

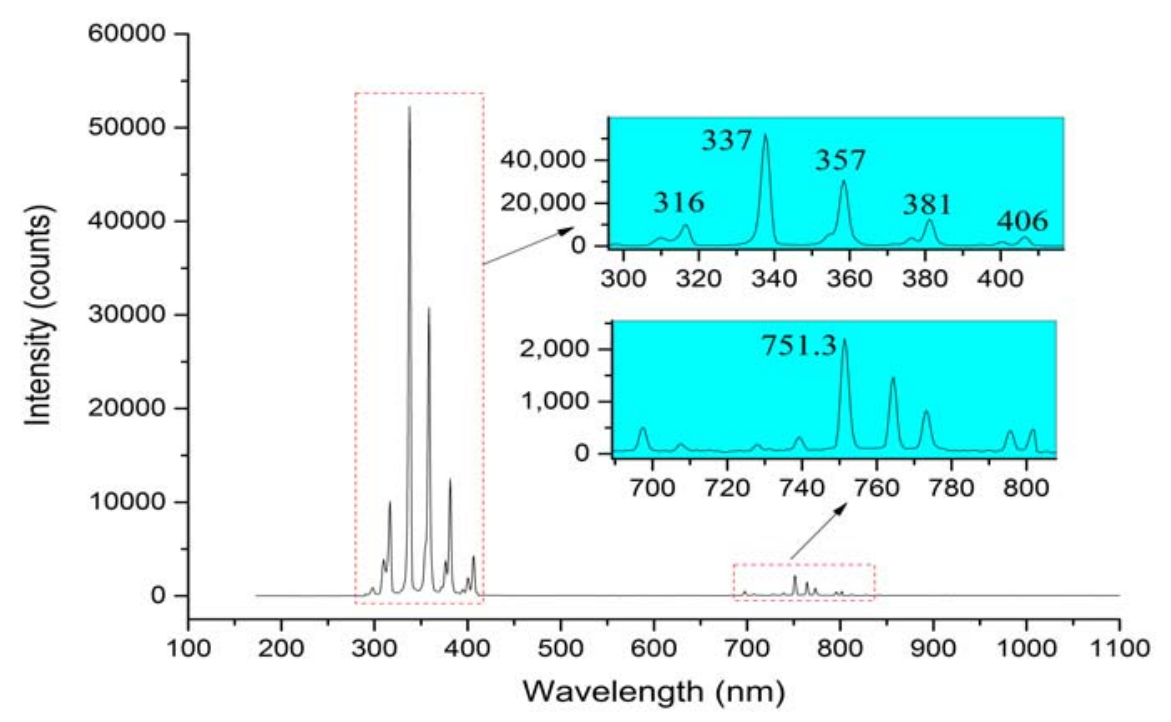

Figure 3: The Spectrum of Shortwave Emission from Ar -N2 Gas Triboplasma Discharge

\section{Wind-Driven Shortwave Emission Device}

Based on the experiment of by means of the reciprocating cycle of mechanical friction, a wind-driven shortwave emission device has been fabricated. The double-cylindrical shape of quartz glass tube was designed to ensure rotating motion by wind-driven(see Fig 4(a)), and quartz glass with high light transmittance can reduce the absorption of shortwave light. Figure 4(b) shown the rotating motion rubbing part, PTFE brick(2) against on the double-cylindrical quartz glass tube(1) was fixed on the rotating part(4) driven by center shaft(5), and the normal force between PTFE and glass was applied and adjusted by loading spring(3) fixed on the support rod(3). The rubbing unit with four rubbing pairs was structured to ensure the high intensive and continue shortwave emission for light trapping agricultural pests. 


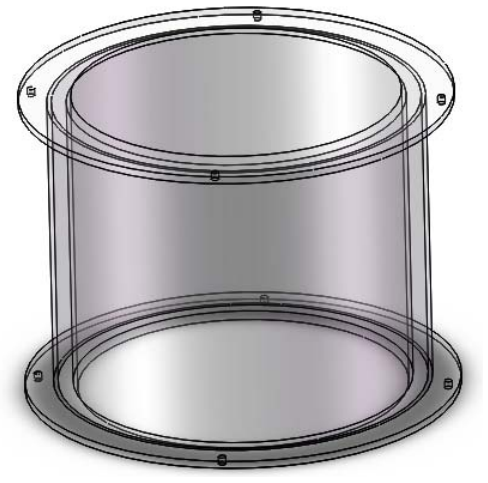

(a)

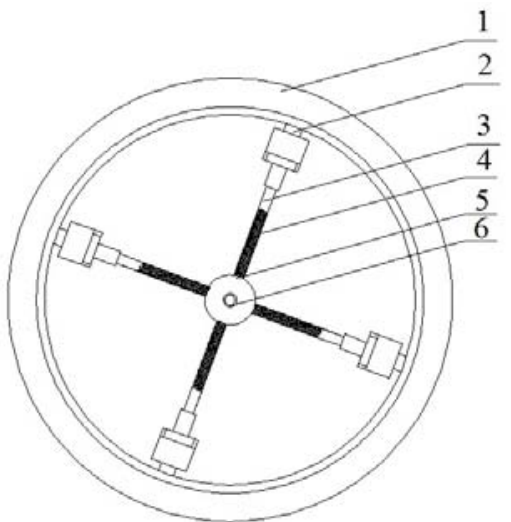

(b)

Figure 4(a) A double-cylindrical quartz glass tube. (b) rotating motion rubbing part

1. Double-cylindrical quartz glass tube; 2. PTFE part; 3. Support rod; 4. Loading Spring; 4. Rotating Part; 5.Center Shaft

As shown in Figure 5, a total structure of shortwave emission device was constituted according to wind turbine driving style and light emitting intensity requirement. The rotating motion rubbing part(4) was directly driven by vertical shaft wind turbine(1) through a vertical center shaft(2), which can ensure the shortwave emission device self-powered. The support pole(6) can be adjusted to lift or lower the height of device to adapt the requirements of crop field or to harvest much wind energy.The shortwave emission device can supply a high intensity of light emission. So it satisfy a basic threshold requirement for light source of trapping agricultural pest insects.

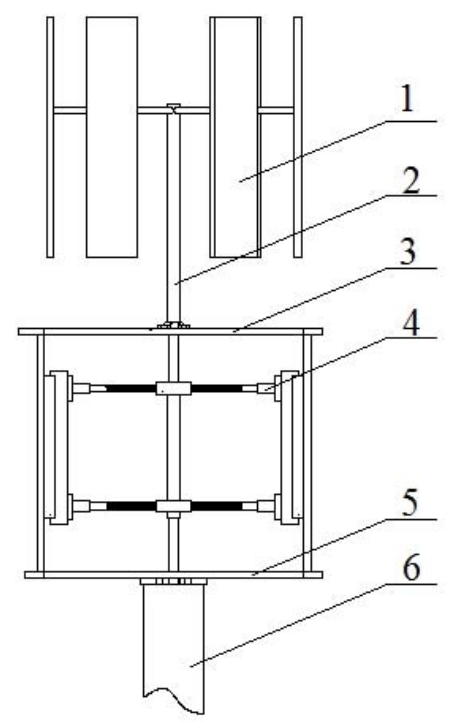

Figure 5 Total Structure of Wind-Driven Shortwave Emission Device

1.Vertical Wind Turbine; 2. Vertical Center Shaft ;3. Top support Plate; 4.Rotating Motion Rubbing Part; 5. Bottom Support Plate; 6.Support Pole

\section{Summary}

The intensity of shortwave emission from triboplasma discharge induced by triboelectri-fication using the reciprocating cycle of mechanical friction between PTFE and quartz glass tube containing $\mathrm{Ar}-\mathrm{N}_{2}$ mixed gas has be investigated.

The result shows the high intensity emission of ten thousand counts in the full near UV spectrum 
from $300 \mathrm{~nm}$ to $400 \mathrm{~nm}$, even the peak value wavelength more than fifty thousand counts. It also prove shortwave emission from Ar -N2 gas triboplasma discharge can be used as special light source.

The double-cylindrical shape of quartz glass tube is designed to form the shortwave light emission device using wind-driven mechanical friction to correlate the triboelectrification with triboplasma discharge, This self-powered device driven by wind can be useful for controlling agricultural pest insect in the vast field without extra energy supply.

\section{Acknowledgement}

The project is supported by the National Natural Science Foundation of China(NSFC),grant number :51375482. We keenly express our thank for NSFC support.

\section{References}

[1] Jeffrey I Z, Gordon E H, James E S, et al. Triboluminescence of sugars. The Journal of Physical Chemistry, 1976, 80(3): 248-249.

[2] Takashi M, Michael C, Roland B. Forces, charges, and light emission during the rupture of adhesive contacts. J. Phys. D: Appl, 2007, 102(10):103509-103514.

[3] Nakayama, K. Hashimoto, H. Wear.Triboemission of charged particles and photons from wearing ceramic surfaces in various hydrocarbon gases. (1995).185:183-188.

[4] Nakayama, K.Surface \& Coatings Technology. Triboemission of electrons, ions, and photons from diamond like carbon films and generation of tribomicroplasma. 2004,599- 604.

[5] Nakayama K, Nevshupa RA. Plasma generation in a gap around a sliding contact. J.Phys. D: Appl, 2002, 35(35):L53-L56(4).

[6] Nakayama K. Triboplasma Generation and Triboluminescence: Influence of Stationary Sliding Partner. Tribology Letters, 2010, 37(2):215-228.

[7] Chang B H,Zhang C,Tan J J, et al . Triboelectrification induced UV emission from plasmon discharge. Nano Research, 2015, 8(1):219-226.[8] Wang, S.H.; Xie, Y. N,.Freestanding triboelectric-layer-based nanogenerators for harvesting energy from a moving object or human motion in contact and non-contact modes. Adv. Mater. 2014, (26):2818-2824.

[9] Diaz AF, RM Felix-Navarro. A Semi-quantitative Tribo-electric Series for Polymeric Materials: the Influence of Chemical Structure and Properties. Journal of Electrostatics, 2004, 62(4):277-290.

[10] Nakayama, K., Nevshupab, R. A.Vacuum. Effect of dry air pressure on characteristics and patterns of tribomicroplasma. 2004,(74): 11-17. 